\title{
Dibujando la realidad usando las Isometrías en el plano bidimensional ${ }^{1}$
}

\section{Drawing Isometric reality using the two-dimensional plane}

\section{Desenho realidade isométrica utilizando o plano bidimensional}

Recibido: mayode 2013

Aceptado: agosto de 2013
Geral Stivens Galán García ${ }^{2}$

Yeimy Rodríguez García ${ }^{3}$

\section{Resumen}

El proyecto de innovación pedagógica se centra en la enseñanza-aprendizaje de las isometrías en el plano bidimensional, en los grados sexto y séptimo; se enfatiza en su utilidad para el desarrollo del arte gráfico, específicamente las teselaciones. Se exponen los pasos a seguir para la construcción de una unidad de teselación, haciendo uso de imágenes dadas, y el software gratuito Geogebra.

Palabras clave: Innovación pedagógica; matemáticas escolares; geometría; formas geométricas; isometrías; teselado; Educación Matemática y otras disciplinas; diseño artístico.

\section{Abstract}

The pedagogical innovation project focuses on the teaching and learning of the two-dimensional plane isometries, in grades six and seven, is emphasized in its use for the development of graphic art, specifically the tessellations. It outlines the steps for building a tessellation unit, using images given, and free software GeoGebra.

Keywords: Innovation pedagogical school mathematics, geometry, shapes, isometrics, tessellation, Math Education and other disciplines, artistic design.

\section{Resumo}

O projeto de inovação pedagógica centra-se no ensino e aprendizagem das isometrias plano bidimensional, em notas seis e sete anos, é enfatizada na sua utilização para o desenvolvimento de arte gráfica, mais especificamente as pavimentações. Ele descreve as etapas para a construção de uma unidade de tessellation, o uso de imagens dadas, e GeoGebra software livre.

Palavras-chave: Inovação matemática pedagógicos escolares, geometria, formas, isométricos, tessellation, Educação Matemática e outras disciplinas, desenho artístico.

1 Artículo de Investigación.

2 Universidad Distrital Francisco José de Caldas. Bogotá, Colombia. Contacto: geraldstevengg@gmail.com

3 Universidad Distrital Francisco José de Caldas. Bogotá, Colombia. Contacto: yeimy.rodriguez.garcia@gmail.com 


\section{Introducción}

El proyecto de innovación se formula teniendo en cuenta las políticas educativas planteadas por el ministerio de educación nacional, para el grado sexto y séptimo, en lo que respecta al pensamiento espacial y sistemas geométricos, donde se indica que los alumnos deben predecir y comparar los resultados de aplicar transformaciones rígidas sobre figuras bidimensionales (traslaciones, rotaciones y reflexiones), en situaciones matemáticas y el arte. Surge el interés por desplegar una propuesta pedagógica para la comprensión de dichas transformaciones haciendo uso de la visualización de figuras geométricas en contextos reales, y modificando figuras bidimensionales en el plano, haciendo uso del software Geogebra en didáctica de la geometría.

Se parte del establecimiento de correspondencias entre la construcción de una unidad de teselación y los referentes teóricos que dan cuenta de ella, aquí se destaca a Van Hiele, citado por Alsina (2000), y sus niveles de desarrollo del pensamiento geométrico, y Pollack, citado por Alsina (2000) con las etapas de la modelización; sin embargo estas fases son aplicables después de tener ciertos conocimientos geométricos incluyendo las transformaciones en el plano; se sugieren unos ejercicios específicos que permiten conocer los elementos geométricos implícitos dentro de la construcción de una imagen bidimensional cualquiera en el programa Geogebra (Pastor A. y Gutiérrez A., 1996), y el diseño y formulación de una situación fundamental que permita realizar las modificaciones de una figura respecto a un objeto de la realidad (Brousseau, 1986), de forma tal que el resultado de dichas modificaciones conlleve a una figura que se caracterice por poseer una o más isometrías (teselado).

A continuación se caracteriza la construcción de una unidad de teselación a partir de una imagen real, y la modificación de una figura regular; se propone como situación fundamental generar una figura bidimensional irregular que recubra por completo una superficie: 1) identificar un objeto de interés (felinos, tiburones y mantas raya), 2) conseguir una imagen referente al objeto establecimiento de una figura geométrica bidimensional que se acomode a las necesidades de la posterior modificación (cuadrado y rectángulo); por ejemplo si se quiere dibujar un pez, podría ser muy conveniente optar por el uso de un cuadrilátero o un triangulo; 4) modificación geométrica por partes (véase figura 2), en este punto es donde los conocimientos geométricos previos, y la situación fundamental se ponen en juego, 5) en esta fase se hace la comparación de la figura resultante con otra igual, determinando si esta contiene isometrías $\mathrm{y}$ además puede cubrir una superficie por completo ante su iteración.

\section{Marco teórico}

Este proyecto tiene en cuenta las ocho etapas de la modelización según Pollack citado por Alsina (2000), que son: 1) Identificación de algo del mundo real que se quiera conocer. 2) Selección de objetos que son importantes en la cuestión del mundo real, e identificación de la relación entre ellos. 3) Selección de objetos que se ignorarán; se obtiene una versión idealizada del objeto. 4) Traducción de la versión idealizada a términos formales; formulación matematizada; modelo geométrico. 5) Identificación de apartados de la geometría que pueden ser relevantes para el modelo y consideración de sus posibles contribuciones. 6) Uso de métodos geométricos e ideas para obtener resultados. 7) Traslado de los resultados a la cuestión inicial; para obtener una teoría sobre lo idealizado. 8) Verificación del resultado en la realidad, si es práctico, razonable, y consecuencias aceptables.

Lo anterior se articula con las fases de aprendizaje de Van Hiele caracterizadas por Pastor A. y Gutiérrez A. (1996), donde se destacan las etapas de información y orientación rígida; el maestro obtiene información acerca de los conocimientos previos de sus estudiantes, y los alumnos reciben ejercicios y problemas referentes a la construcción del nuevo concepto, aquí se hace referencia a los conocimientos pre-requeridos para el desarrollo de la secuencia, y se tiene en cuenta la teoría de situaciones didácticas de Brousseau (1986).

Finalmente para la construcción de la imagen con transformaciones rígidas, nuevamente se toman en consideración los planteamientos de Van Hiele, 
donde los dos primeros niveles de desarrollo del pensamiento geométrico, se acomodan a las fases propuestas para la construcción de la unidad de teselación:

Visualización: Percepción las figuras como un todo global; los objetos que son razonados son clases de figuras reconocidas visualmente como de "la misma forma”, o en este caso la identificación del objeto que se desea dibujar a partir de múltiples imágenes que pueden ser generalizadas en una sola (idealizadas).

Análisis: Mediante el conocimiento de las componentes de las figuras, y sus propiedades básicas, se podría realizar cualquier modificación necesaria a la figura inicial para lograr la imagen deseada; se piensa en términos del conjunto de propiedades asociadas a la figura (MEN, 1998).

\section{Conclusiones}

El proceso de construcción de una imagen de un objeto real, haciendo uso de las isometrías y buscando su comprensión, puede ser bastante largo y complicado, ante lo cual se puede inferir que las ideas citadas aquí no sean suficientes para lograr tal objetivo, sin embargo y tratándose de un proyecto de innovación pedagógica, estos referentes son considerados como la base fundamental para desarrollar el proyecto, de modo que en la medida en que se consolide, se excluirán o incluirán ideas de nuevos autores.

\section{Referencias}

Alsina, C. (2000). Geometría y realidad. Universidad Politécnica De Cataluña, España.

Brousseau, G. (1986). Fondements et méthodes de la didactiques des mathématiques. Recherches en didactique des mathématiques, 7, 2, P. 33115. (Fundamentos y métodos de la didáctica de la matemática. Traducción de Dilma Fregona y Facundo Ortega). Argentina.

MEN. (1998). Lineamientos curriculares para el área de matemáticas. Bogotá: Magisterio.

Pastor, A. y Gutiérrez, A.(1996). El grupo de las isometrías del plano. Madrid: Síntesis. 